\title{
Negative Regulation of Violacein Biosynthesis in Chromobacterium violaceum
}

\begin{abstract}
Giulia Devescovi ${ }^{1}$, Milan Kojic ${ }^{2}$, Sonia Covaceuszach ${ }^{3}$, Miguel Cámara ${ }^{4}$, Paul Williams ${ }^{4}$, Iris Bertani ${ }^{1}$, Sujatha Subramoni ${ }^{1 \dagger}$ and Vittorio Venturi ${ }^{1 *}$

${ }^{1}$ International Centre for Genetic Engineering and Biotechnology, Trieste, Italy, ${ }^{2}$ Laboratory for Molecular Microbiology, Institute of Molecular Genetics and Genetic Engineering, University of Belgrade, Belgrade, Serbia, ${ }^{3}$ Consiglio Nazionale delle Ricerche, Istituto di Cristallografia, U.O.S di Trieste, Trieste, Italy, ${ }^{4}$ Centre for Biomolecular Sciences, School of Life Sciences, University of Nottingham, Nottingham, UK
\end{abstract}

In Chromobacteium violaceum, the purple pigment violacein is under positive regulation by the $\mathrm{N}$-acylhomoserine lactone $\mathrm{Cvil} / \mathrm{R}$ quorum sensing system and negative regulation by an uncharacterized putative repressor. In this study we report that the biosynthesis of violacein is negatively controlled by a novel repressor protein, VioS. The violacein operon is regulated negatively by VioS and positively by the Cvil/R system in both $C$. violaceum and in a heterologous Escherichia coli genetic background. VioS does not regulate the Cvil/R system and apart from violacein, VioS, and quorum sensing regulate other phenotypes antagonistically. Quorum sensing regulated phenotypes in C. violaceum are therefore further regulated providing an additional level of control.

Keywords: Chromobacterium violaceum, VioS, Cvil/R quorum sensing, regulation, violacein, chitinase activity, protease activity

\section{INTRODUCTION}

Many Gram-negative bacteria regulate cell density dependent behavior by producing and sensing $\mathrm{N}$-acylhomoserine lactone (AHL) signal molecules by a process called quorum sensing (QS; Fuqua et al., 1994). A canonical AHL-dependent QS system is composed of two proteins respectively belonging to the LuxI and LuxR protein families (Fuqua et al., 1996). Typically, AHLs are produced by an AHL synthase (LuxI homolog) and sensed at a threshold concentration due to increase in cell population density by an AHL-binding regulator (LuxR homolog) which then affects transcription of target genes (Fuqua and Greenberg, 2002). AHL QS regulates many phenotypes that impact on bacterial community or group behaviors including the expression of secreted enzymes, antibiotic and exopolysaccharide production, biofilm formation, conjugation, symbiosis, and virulence (Fuqua and Greenberg, 2002; Loh et al., 2002; Von Bodman et al., 2003; Waters and Bassler, 2005).

Chromobacterium violaceum is a betaproteobacterium found in a variety of soil and aquatic habitats causing infrequent but fatal mammalian infections (Brazilian National Genome Project, 2003). Two C. violaceum strains (ATCC31532 and ATCC12472) possess an AHL QS system and surprisingly they produce and respond to different AHLs (McClean et al., 1997; Morohoshi et al., 2008). The AHL QS system of C. violaceum ATCC12472 is encoded by the genetically linked $c v i I$ and $c v i R$ genes producing and responding with highest affinity to $\mathrm{N}$-decanoyl-L-homoserine lactone (C10-HSL). CviR therefore binds to C10-HSL with highest affinity (Morohoshi et al., 2008; Swem et al., 2009) and the cviI AHL synthase is under positive feedback regulation by 
C10-HSL-CviR (Stauff and Bassler, 2011). The CviI/CviR QS system of C. violaceum ATCC12472 is important for virulence as revealed by loss of pathogenicity in a C. elegans infection model in the presence of an antagonistic ligand for CviR instead of C10-HSL (Swem et al., 2009). In contrast, a much earlier report (McClean et al., 1997) demonstrated that the AHL signal produced by C. violaceum ATCC31532 is C6-HSL. However, cloning and genetic analysis of this $c v i I / c v i R$ QS system has not been yet been reported in detail.

In C. violaceum, QS regulates (i) the vioA promoter of violacein vioABCDE genes coding for the water insoluble purple pigment violacein (Lichstein and Van De Sand, 1946; McClean et al., 1997), (ii) genes coding for cyanide production and degradation (Durán and Menck, 2001), and (iii) multiple genes the products of which are chitinases (Chernin et al., 1998). Besides the cviI promoter, several other genes are directly regulated by CviR in C. violaceum ATCC12472 and these include genes coding for a putative transcriptional regulator (CV_0577), a guanine deaminase (CV_0578), a chitinase (CV_4240), and a type VI secretion system gene (CV_1432) (Stauff and Bassler, 2011). As in C. violaceum AHL QS regulates the production of the purple pigment violacein; this has allowed the convenient use of this bacterium as an AHL biosensor since the AHLnegative biosensor strain CV026 produces violacein only upon the addition of exogenous AHLs with from C4 to C8 acyl side chains (McClean et al., 1997; Steindler and Venturi, 2007).

Regulation of violacein production by QS has been studied in more detail than the other phenotypes as it is an easily discernible and visible trait. Using a combination of mutagenesisbased analysis in C. violaceum ATCC 31532 and experiments in a heterologous Escherichia coli host, the vioA promoter of vioABCDE operon has been shown to be under the direct positive regulation of CviR (McClean et al., 1997; Swem et al., 2009). Comprehensive mutational analysis of the vioA promoter has also enabled the identification of a CviR binding site (Stauff and Bassler, 2011). Interestingly, the level of violacein produced by wild type C. violaceum ATCC12472 is much higher than that of wild type C. violaceum ATCC31532 (McClean et al., 1997). Furthermore, a violacein repressor has been reported and inactivated by transposon mutagenesis in two independent studies in C. violaceum ATCC31532 giving rise to mutants with considerably higher violacein production (McClean et al., 1997; Swem et al., 2009). In addition, the Chromobacterium AHL biosensor strain CV026 is a double transposon insertion mutant since single Tn5 insertions in the putative AHL synthase failed to respond to exogenous AHLs unless a second transposon was introduced into the putative repressor locus (McClean et al., 1997). However, the mechanism of violacein regulation by this putative repressor and its regulatory relationship with the $C$. violaceum AHL QS system are not known.

In this study we have examined the regulation of violacein production in C. violaceum ATCC 31532 and characterized its QS system as well as a repressor mutant of this strain with respect to violacein production. We show that the expression of the vioA promoter of the vioABCDE operon is under negative regulation by this novel repressor which we have named VioS. VioS is also involved in the regulation of other AHL QS regulated phenotypes such protease and chitinolytic activity. Furthermore, we provide evidence for direct interference by VioS of QS mediated positive regulation of the vioA promoter in C. violaceum and in E. coli. Finally, we show that VioS functions as a repressor of violacein production in the closely related C. violaceum ATCC12472 when introduced in trans. We propose that VioS is a novel protein that functions to fine-tune the QS regulated phenotype of violacein biosynthesis by regulating vioA promoter expression rather than modulating the regulation of $c v i I / c v i R$ gene expression.

\section{MATERIALS AND METHODS}

\section{Bacterial Strains, Media, and Growth Conditions}

Wild type C. violaceum ATCC 31532, ATCC12472, and CV026 (McClean et al., 1997) and Escherichia coli strains DH5 $\alpha$ and M15 were routinely grown at $30^{\circ} \mathrm{C}$ and $37^{\circ} \mathrm{C}$, respectively, in Luria-Bertani (LB) broth medium (Miller, 1972). When required, antibiotics were added in the following concentrations: ampicillin $100 \mu \mathrm{g} \mathrm{ml}^{-1}$, kanamycin $100 \mu \mathrm{g} \mathrm{ml}^{-1}$, gentamicin $50 \mu \mathrm{g} \mathrm{ml}^{-1}$, tetracyclin $40 \mu \mathrm{g} \mathrm{ml}^{-1}$ for C. violaceum strains and, ampicillin $100 \mu \mathrm{g} \mathrm{ml}^{-1}$, kanamycin $50 \mu \mathrm{g} \mathrm{ml}^{-1}$, gentamycin $20 \mu \mathrm{g} \mathrm{ml}^{-1}$ and tetracyclin $20 \mu \mathrm{g} \mathrm{ml}^{-1}$ for Escherichia coli strains. AHLs used here were obtained from Sigma-Aldrich (St. Louis, MO, USA).

\section{Recombinant DNA Techniques}

DNA manipulations, including digestion with restriction enzymes, agarose gel electrophoresis, purification of DNA fragments, ligation with T4 DNA ligase, transformation of $E$ coli, colony hybridization and radioactive labeling by random priming, were performed as previously described (Sambrook et al., 1989). Plasmids were purified using EuroClone columns (EuroClone S.p.A., Italy). Total DNA from C. violaceum was isolated with the sarkosyl-pronase lysis method (Better et al., 1983). Triparental matings to mobilize DNA from $E$. coli to C. violaceum were carried out with the helper strain E. coli (pRK2013) (Figurski and Helinski, 1979). PCR amplifications were performed on C. violaceum ATCC 31532 genomic DNA using GoTaq Flexi DNA Polymerase (Promega, Madison, WI, USA).

\section{Plasmid Construction}

The plasmids used in this study are listed in Table 1.

The $g f p$ reporter gene was chosen for studying the promoter activities in C. violaceum in order to reduce to the minimum, possible, interference by violacein that can be an issue with the $\beta$-galactosidase assay. A $g f p$ based reporter plasmid was constructed by amplifying the $g f p$ gene, deprived of its promoter, from plasmid pBBR2-GFP (Passos da Silva et al., 2014) using the primers GFPEF and GFPPR. The amplified $g f p$ was then cloned as an EcoRI/PstI fragment in pMP220 vector, generating pMPGFP.

Gene transcriptional fusion plasmids, based on the pMPGFP promoter probe vector, were constructed as follows: the promoter regions of $c v i I, c v i R$, vioA, and vioS genes were amplified from C. violaceum 31532 genomic DNA by using, 
TABLE 1 | Strains, plasmids, and primers used.

\begin{tabular}{|c|c|c|}
\hline $\begin{array}{l}\text { Strains } \\
\text { /plasmids/ } \\
\text { primer }\end{array}$ & Relevant features & $\begin{array}{l}\text { References or } \\
\text { sources }\end{array}$ \\
\hline \multicolumn{3}{|c|}{ C. violaceum STRAINS } \\
\hline $\begin{array}{l}\text { C. violaceum } \\
\text { ATCC31532 }\end{array}$ & WT isolate & \\
\hline $\begin{array}{l}\text { C. violaceum } \\
\text { ATCC12472 }\end{array}$ & WT isolate & \\
\hline CV026 & $\begin{array}{l}\text { Double transposon mutant of ATCC31532, } \\
\text { violacein and AHL negative }\end{array}$ & McClean et al., 1997 \\
\hline MB8 & vioS::Tn5 of C. violaceum ATCC31532; $\mathrm{Km}^{\mathrm{R}}$ & This study \\
\hline MB11 & vioS::Tn5 of C. violaceum ATCC31532; $\mathrm{Km}^{\mathrm{R}}$ & This study \\
\hline 31532VIOS & vioS:: Km of C. violaceum ATCC31532; $\mathrm{Km}^{\mathrm{R}}$ & This study \\
\hline $31532 \mathrm{CVII}$ & cvil::Km of C. violaceum ATCC31532; $\mathrm{Km}^{R}$ & This study \\
\hline 31532CVIR & cviR::Gm of C. violaceum ATCC31532; $\mathrm{Gm}^{\mathrm{R}}$ & This study \\
\hline \multicolumn{3}{|l|}{ PLASMIDS } \\
\hline pRK2013 & $\mathrm{Tra}^{+} \mathrm{Mob}+\mathrm{ColE} 1$ replicon; $\mathrm{Km}^{\mathrm{R}}$ & $\begin{array}{l}\text { Figurski and Helinski, } \\
1979\end{array}$ \\
\hline pGEM2T & Cloning vector; $\mathrm{Amp}^{\mathrm{R}}$ & Promega \\
\hline pMP220 & Promoter probe vector, IncP; $\mathrm{Tc}^{\mathrm{R}}$ & Spaink et al., 1987 \\
\hline pQE30 & Expression vector; $\mathrm{Amp}^{\mathrm{R}}$ & Qiagen \\
\hline pBSIIKS & Cloning vector; $\mathrm{Amp}^{\mathrm{R}}$ & Stratagene \\
\hline pBBRmcs5 & Broad-host-range vector; $\mathrm{Gm}^{R}$ & Kovach et al., 1995 \\
\hline pKNOCK-Km & Conjugative suicide vector; $\mathrm{Km}^{\mathrm{R}}$ & Alexeyev, 1999 \\
\hline pKNOCK-Gm & Conjugative suicide vector; $\mathrm{Gm}^{\mathrm{R}}$ & Alexeyev, 1999 \\
\hline pSUP2021 & Tn5 delivery suicide plasmid; ColE1; $\mathrm{Km}^{\mathrm{R}}$ & Simon et al., 1983 \\
\hline pLAFR3 & Broad-host-range vector, IncP; Tc $^{R}$ & Staskawicz et al., 1987 \\
\hline pCVO7 & $\begin{array}{l}\text { pLAFR3 containing C. violaceum } 31532 \text { DNA; } \\
T_{C}^{R}\end{array}$ & This study \\
\hline pBSCVO7H & $\begin{array}{l}\text { pBSIIIKS carrying a HindllI } 3 \mathrm{~kb} \text { fragment from } \\
\text { CVO7; AmpR }\end{array}$ & This study \\
\hline pBCVO7XN & $\begin{array}{l}\text { pBSIIIKS carrying a Xhol-Notl } 6.35 \text { kb fragment } \\
\text { from CVO7; } \text { Amp }^{R}\end{array}$ & This study \\
\hline pBBVios & pBBRmcs 5 containing Vios ; $\mathrm{Gm}^{\mathrm{R}}$ & This study \\
\hline pKNOCKcvil & Internal cvil fragment cloned in pKNOCK-Km & This study \\
\hline pKNOCKcviR & Internal cviR fragment cloned in pKNOCK-Gm & This study \\
\hline pKNOCKvioS & Internal vioS fragment cloned in pKNOCK-Km & This study \\
\hline pMPGFP & $\begin{array}{l}\text { pMP220 containing the GFPmut3 gene deprived } \\
\text { of its promoter }\end{array}$ & This study \\
\hline pPcvilGFP & cvil promoter cloned in pMPGFP & This study \\
\hline pPcviRGFP & cviR promoter cloned in pMPGFP & This study \\
\hline pPvioAGFP & vioA promoter cloned in pMPGFP & This study \\
\hline pPvioSGFP & vioS promoter cloned in pMPGFP & This study \\
\hline pBBRcviR & cviR cloned in pBBRmcs5 & This study \\
\hline pQE30VioS & vioS cloned in PQE30 & This study \\
\hline pPvioA220 & vioA promoter cloned in pMP220 & This study \\
\hline pPcepl220 & cep/ promoter cloned in pMP220 & This study \\
\hline pscR2 & $\begin{array}{l}\text { pQF50 vector expressing the B. cepacia cepR } \\
\text { gene }\end{array}$ & Aguilar et al., 2003 \\
\hline pMP77 & Promoter probe vector; IncQ; CmR & Spaink et al., 1987 \\
\hline pMPCviRLacZ & cviR translational fusion & This study \\
\hline pMPVioALacZ & vioA translational fusion & This study \\
\hline \multicolumn{3}{|l|}{ PRIMERS } \\
\hline Primers name & Sequence & Source \\
\hline cvilBF & GGATCCCCGTAGGCAAAGAACTAA & This study \\
\hline cvilER & GAATTCTTGTGTCTGAACGCCA & This study \\
\hline cviRBF & GGATCCCCGAAACTCATCCAAAAA & This study \\
\hline cViRER & GAATTCGTTGATGGGTTTCGAGAT & This study \\
\hline vioABF & CGGATCCGTGTTGCATITCTCAAATGG & This study \\
\hline vioAER & GGAATTCGAAGAGTGCTTCATCACGA & This study \\
\hline vioSBF & GGATCCGCCCAAAGCCAGACTA & This study \\
\hline vioSER & GAATTCTGAACGGCACGATTGA & This study \\
\hline GFPEF & GGAATTCAAGAGGAGAAATTAAGATG & This study \\
\hline GFPPR & ACTGCAGTCAGCTAATTAAGCTTATT & This study \\
\hline
\end{tabular}

(Continued)
TABLE 1 | Continued

\begin{tabular}{lll}
\hline $\begin{array}{l}\text { Strains } \\
\text { lplasmids/ } \\
\text { primer }\end{array}$ & Relevant features & $\begin{array}{l}\text { References or } \\
\text { sources }\end{array}$ \\
\hline $\begin{array}{l}\text { vioA220KF } \\
\text { vioA220XR }\end{array}$ & AGGTACCGTGTTGCATTCTC & This study \\
cepl220EF & GACTAGAGAAGAGTGCTTCAT & This study \\
cepl220XR & TCTAGAGCATGGTGTCCTCGGATT & This study \\
cviRPROMF_Xba & TCTAGAGCCGAAACTCATCCAAAA study \\
cviR2R_Bglll & AGATCTGGGCGTAGTTTCCTCATGT & This study \\
vioAPROMF_Xba & GTCTAGAAATGGAAAGCCTGTCACT & This study \\
vioAR3_BamHI & AGGATCCTCTGCATGTCGAAAT & This study \\
VioSBFW & AGGATCCCCTTGCATCACCCGCAGT & This study \\
VioSHR & GAAGCTTTACGAGGCGGGGTTAGA & This study \\
cviRHF & CAAGCTTCAAGGAAGACTCGCTCAT & This study \\
cviRXR & GTCTAGATCATTCGTTCGCTACGGT & This study \\
KncvilBF & AGGATCCAGGCTATTGGTGCC & This study \\
KNcvilKR & AGGTACCAGCCGGCGGTACAT & This study \\
KNcviRF & CCAGAACCAGATCCAGCG & This study \\
KNcviRR & GATGGACAGGATGCTGCCG & This study \\
KNvioSKF & AGGTACCCGGCTGCACGAAGC & This study \\
KNvioSBR & AGGATCCCAGGCAAGCCAGC & This study \\
\hline
\end{tabular}

respectively, the primers cviIBF and cviIER (cviIPROM; 337bp), cviRBF, and cviRER (cviRPROM; 277-bp), vioABF and vioAER (vioAPROM; 328-bp), vioSBF, and vioSER (vioSPROM; 196-bp). The amplified fragments were cloned in pGEM-T Easy vector (Promega, Madison, WI, USA), sequenced and then excised as $B a m H I / E c o R I)$ fragments and cloned into the $B g l \mathrm{II} / \mathrm{EcoRI}$ sites in pMPGFP obtaining pPcviIGFP pPcviRGFP, pPvioAGFP, and pPvioSGFP constructs. The vioA promoter was also amplified with primers vioA220KF and vioA220XR and cloned as a $K p n I / X b a I$ fragment into the corresponding restriction sites of the promoter probe vector pMP220, obtaining pPvioA220 construct. The cepI promoter was amplified with primers cepI220EF and cepI220XR and cloned as a EcoRI/XbaI fragment in pMP220 giving pPcepI220. The vioS gene with its promoter was cut out from the $\mathrm{pBSCVO} 7 \mathrm{H}$ construct as a SnaBI/XbaI fragment and cloned into the corresponding restriction sites of pBBRmcs5 to generate pBBRvioS. The vioS gene was also amplified from C. violaceum 31532 genomic DNA using the primers VioSBFw and VioSHR and cloned into the BamHI/HindIII restriction sites of $\mathrm{pQE} 30$ vector to generate $\mathrm{pQE} 30 \mathrm{VioS}$. The $c v i R$ gene was amplified from C. violaceum 31532 genomic DNA using primers cvRHF and cvRXR and inserted downstream of the lac promoter in $\mathrm{pBBRmcs} 5$ linearized with HindIII and $\mathrm{XbaI}$ restriction enzymes. The fidelity of all of the constructs described was verified by DNA sequencing (Macrogen, Europe).

Translational fusions were constructed as follows: the $5^{\prime}$ region of the $c v i R$ DNA sequence, containing the promoter and coding sequences for the first 98 amino acids was amplified from $C$ violaceum 31532 genomic DNA by using the primers cviRPROMFXba and cviR2RBglII. The amplified fragment was then cloned in frame upstream from the lacZ gene and then the whole construct was transferred into the pMP77 vector generating pMPCviRLacZ. Similarly, the $5^{\prime}$ region of the vioA gene, containing the promoter and coding sequences for the 
first 49 amino acids was amplified by using the primers vioAPROMFXba and vioAR3BamHI, cloned in-frame upstream the lac $Z$ gene and transferred to the pMP77 plasmid giving pMPVioAlacZ.

\section{Genomic Mutant Bank and Cosmid Gene Bank Construction and Screening}

A Tn5 genomic mutant library of C. violaceum ATCC31532 was created using pSUP2021, as previously described (Simon et al., 1983). Approximately 5,000 mutants were screened for the presence of violacein hyperproducer mutants by identifying colonies that showed purple coloration in contrast to the pale colonies of the C. violaceum ATCC31532 wild type. Two mutants were isolated and the genomic regions flanking the Tn5 insertions were amplified by arbitrary PCR technique (O'Toole and Kolter, 1998) and sequenced. The two mutants were designated as MB8 and MB11 respectively. A genomic bank (cosmid library) of C. violaceum ATCC31532 was constructed as follows. Briefly, C. violaceum 31532 genomic DNA was partially digested with EcoRI and ligated into pLAFR3 cosmid vector. The constructs obtained were introduced into E. coli cells using Gigapack III XL-4 packaging kit as recommended by the supplier (Stratagene-Agilent, Santa Clara, CA, USA). The genomic bank was then screened using the flanking DNA (obtained by arbitrary PCR on mutant colonies MB8 and MB11), as probes. Three cosmids were isolated which showed the same restriction pattern. Cosmid pCVO7 was chosen and subcloned in pBSIIKS generating two overlapping constructs: $\mathrm{pBCVO} 7 \mathrm{H}$ (containing a 3-kb HindIII fragment) and pBCVO7XN (containing a 6,350-bp XhoI-NotI fragment).

\section{Construction of $31532 \mathrm{CVII}, 31532 \mathrm{CVIR}$, and 31532VIOS}

The three additional mutants, 31532CVII, 31532VCIR, and 31532 VIOS were generated using the suicide vectors from the pKNOCK series (Alexeyev, 1999). To generate 31532cviI, an internal fragment (209-bp) of the cviI gene was PCR amplified using the primers KNcviIBF and KNcviIKR and cloned as a $B a m H I-K p n I$ fragment into the corresponding sites of pKNOCK$\mathrm{Km}$ resulting in pKNOCKcviI. In order to generate 31532CVIR, an internal fragment of $c v i R$ (327-bp) was amplified with the primers KNcviRF and KNcviRR, blunted and cloned into pKNOCK Gm digested with the SmaI restriction enzyme, yielding pKNOCKcviR. Finally, to obtain 31532VIOS, an internal fragment of vioS (187-bp) was amplified with primers KNvioSKF and KNvioSBR and cloned as a KpnI-BamHI fragment in the corresponding sites of $\mathrm{pKNOCK}-\mathrm{Km}$ giving $\mathrm{pKNOCKvioS}$. The pKNOCK constructs obtained were transferred to C. violaceum ATCC31532 via tri-parental mating and the knock-out mutants were verified by PCR analysis and sequencing. The 31532 VIOS was altered in growth rate and behaved like the parent wild-type strain.

\section{Extraction and Quantification of AHLs}

C. violaceum strains were grown overnight in $20 \mathrm{ml}$ of $\mathrm{LB}$ medium. The cells were pelleted at 5,000 $\mathrm{g}$ for $15 \mathrm{~min}$. The cell free supernatants were filtered (using $0.45 \mu \mathrm{m}$ filters; Millipore) and extracted twice with an equal volume of ethyl acetate containing $0.1 \% \mathrm{v} / \mathrm{v}$ acetic acid. The organic phases were collected, dried to completeness and re-suspended in $50 \mu \mathrm{l}$ of ethyl acetate. To quantify the amounts of C6-HSL produced by the 31532 wild type strain, MB8, MB11, and 31532VIOS, the constructs pPvioA220 and pBBRcviR were used to constitute a CviR-based sensor regulating its target promoter vioA in the heterologous $E$. coli M15 system. In order to generate a calibration curve, different concentrations $(0 ; 0.01 ; 0.05 ; 0.1 ; 0.5$; $1 \mu \mathrm{M}$ ) of C6-HSL were added to $10 \mathrm{ml}$ to each of the sensor strains. The cultures were grown for $6 \mathrm{~h}$ and $\beta$-galactosidase activity was determined. To quantify the AHLs produced by each $C$. violaceum strain, the experiment was repeated by adding $10 \mu \mathrm{l}$ of an AHL extract obtained from each strain to the sensor.

\section{$\beta$-Galactosidase and GFP Quantification Assays}

$\beta$-galactosidase activities were determined essentially as described by Miller (1972), with the modifications of Stachel (Stachel et al., 1985). Each experiment was performed in triplicate. GFP fluorescence in the stationary phase of the bacterial cultures was determined in a Perkin Elmer EnVision Multilabel Reader that was set to an excitation wavelength of $485 \mathrm{~nm}$ and an emission wavelength of $510 \mathrm{~nm}$.

\section{Exoenzyme Activity}

To assess protease activity, C. violaceum strains were grown to stationary phase and $2 \mu \mathrm{l}$ of culture was spotted onto M9 agar containing $2 \%$ dry milk, as the only carbon source. Zones of activity were measured after $36 \mathrm{~h}$. For chitinase activity, the same protocol was followed and cultures were spotted onto M9 agar containing $0.2 \%$ colloidal chitin (Ahmadian et al., 2007).

\section{RESULTS}

\section{The AHL QS System of C. violaceum ATCC31532}

The unequivocal chemical identification of C6-HSL from culture supernatants of C. violaceum ATCC 31532 and the selection of a Tn5 transposon mutant with an insertion in a putative luxI orthologue demonstrated the presence of an AHL QS system in this organism (McClean et al., 1997). To isolate the locus encoding this system, a Pst genomic library of this strain was constructed in pUC18. The library was introduced into the AHL biosensor strain E. coli (pSB401) (Winson et al., 1998) and the recombinant colonies screened for the production of bioluminescence using a photon-imaging camera as previously described (Swift et al., 1997). A recombinant clone (pMW50) able to induce light production in the biosensor strain was identified as a highly bioluminescent colony. Expression of pMW50 in E. coli, was able to restore violacein production when crossstreaked against the AHL sensor strain C. violaceum CV026 (McClean et al., 1997) suggesting the presence of an AHL synthase in this recombinant clone. Sequence analysis of the 
$6 \mathrm{~Kb}$ PstI insert from pMW50 revealed the presence of two convergent open reading frames overlapping by $74 \mathrm{bp}$ which were named $c v i R$ and $c v i I$ as their predicted amino acid sequences were homologous to the LuxI/LuxR family of QS genes. Solvent extraction of culture supernatants from E. coli harboring pMW50 followed by LC-MS/MS analysis revealed the presence of C6HSL (data not shown). No other AHLs were detected from these extracts indicating that $c v i I$ is responsible for the synthesis of this AHL.

\section{Violacein Biosynthesis Is Negatively Regulated by VioS}

Violacein production by C. violaceum is regulated by QS via AHLs signal molecules (McClean et al., 1997; Morohoshi et al., 2008). We have previously shown that violacein production is stringently negatively regulated since we obtained a Tn5 insertion mutant that strongly overproduced violacein in the $C$. violaceum ATCC31532 genetic background (McClean et al., 1997; Table 1). This transposon was localized to a gene coding for a protein of unknown function homologous to CV_1055 of the sequenced genome of C. violaceum ATCC12472 demonstrating that violacein is very tightly regulated (Swem et al., 2009). To further investigate the regulation of this phenotype and to make sure that no other loci was involved in this negative regulation, we constructed a Tn5 mutant library of C. violaceum ATCC31532 and screened for more mutants that overproduced violacein as described in the Materials and Methods. Two mutants, named MB8 and MB11 were identified in the screen and the location of the Tn5 insertion site in both mutants was also located in the CV_1055 gene homolog from C. violaceum ATCC12472 but in the putative promoter region; the Tn5 in mutant MB8 is located nearer to the ATG of the putative ORF whereas MB11 is further away (Figure 1A). We have now named the hypothetical protein encoded by this gene as VioS (Figure 1A). This predicted protein (138 amino acids; 15 $\mathrm{kDa}$ approximately) showed $91 \%$ identity and 94\% similarity to a hypothetical protein from Pseudogulbenkiana ferrooxidans and $85 \%$ identity and $90 \%$ similarity to the hypothetical protein encoded by CV_1055 from C. violaceum ATCC12472 respectively. Conserved domain analysis of VioS amino acid sequence revealed the presence of a domain of unknown function annotated as DUF1484 spanning 32-138 amino acids (8.35e-03) that is exclusively found in bacteria belonging to the betaproteobacteria.

Both MB8 and MB11 transposon mutants exhibited increased violacein production in contrast to the pale white color of C. violaceum ATCC31532 wild type (Figure 1B). Mutant MB11 displayed a much stronger violet color compared with MB8 indicating that the transposon insertion in MB11 resulted in greater violacein production. As neither transposon insertion was located in the putative structural gene, an insertion mutant in the putative vioS ORF was generated (designated as 31532VIOS) as described in the Materials and Methods. This mutant showed violacein overproduction similar to MB8 (Figure 1B). Complementation of mutants MB8, MB11, and 31532VIOS with a plasmid construct containing full length vioS and flanking upstream DNA restored violacein production in all the mutants to wild type levels (Figure 1B). These results strongly suggest a role for VioS in the negative regulation of violacein biosynthesis in C. violaceum ATCC31532.

\section{VioS and CviR Regulate Violacein Biosynthesis in Opposite Ways}

Since the studies using the transposon insertion mutants described above clearly support a role for VioS in the negative
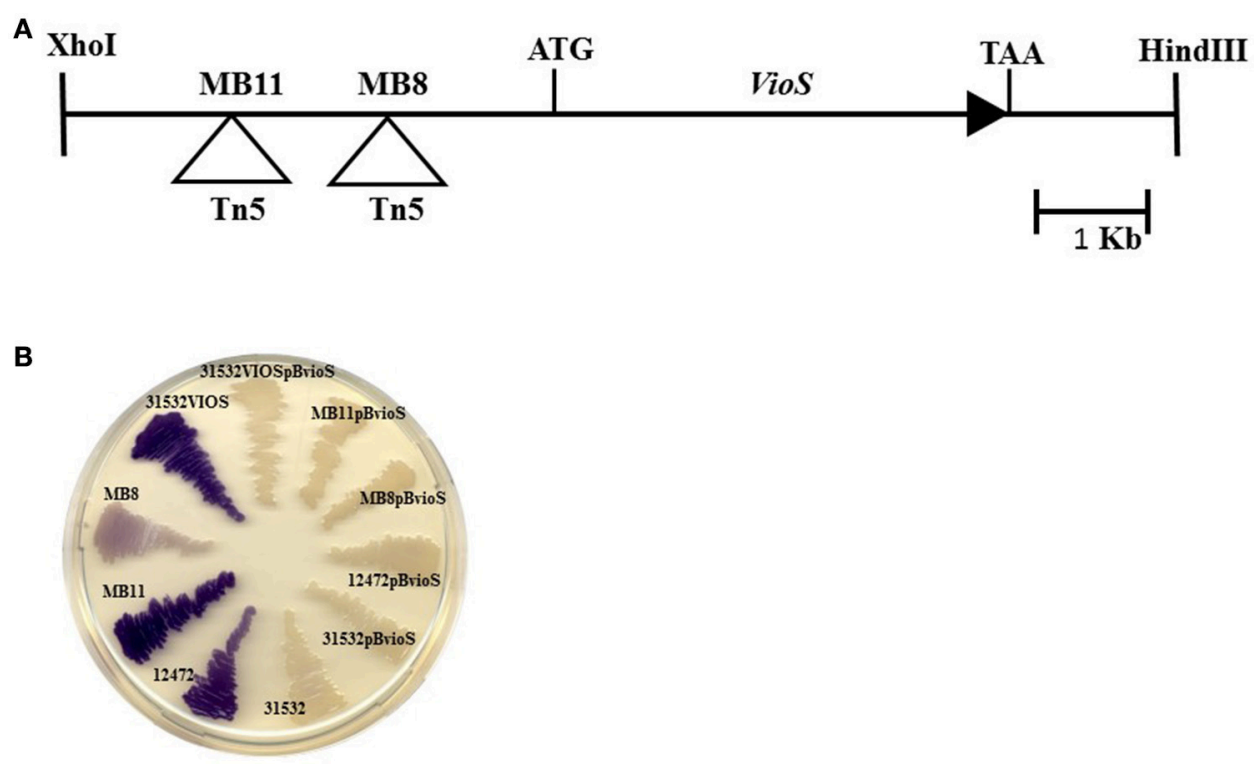

FIGURE 1 | (A) Genomic organization of the vioS locus. The Tn5 insertions in mutants MB8 and MB11 are indicated. (B) Production of violacein in C. violaceum wt strains ATCC31532 and ATCC12472, 31532VIOS mutants (MB8; MB11;31532VIOS), and mutants complemented with pBBVioS, containing full length vioS. 
regulation of violacein production, which conversely is positively regulated by the CviI/R QS system, we sought to determine whether VioS interacted with the QS system. Consequently we investigated whether VioS influenced the expression of the CviI/R system which could then result in violacein de-regulation. We first determined the AHL levels produced by the wild type, MB8, MB11, and 31532VIOS strains as described in the Materials and Methods. Using a calibration curve derived by a CviI/R AHL biosensor constructed here, we found that all strains produced similar AHL levels production corresponding to a C6HSL concentration of approximately $0.5 \mu \mathrm{M}$ (data not shown). The transcriptional levels of the QS genes using cviI::gfp and $c v i R:: g f p$ plasmid transcriptional fusions were determined and the results showed that the $c v i I$ and $c v i R$ genes are expressed at comparable levels in the wild type, the vioS mutants and complemented strains (Figures 2A,B). To determine whether the CviI/R QS system modulated vioS expression, assays were carried out to measure the levels of a plasmid-borne vioS::gfp transcriptional fusion in the wild type, $c v i I$ and $c v i R$ mutants. The expression of vioS was similar in all of the strains examined (data not shown). These results indicate that VioS does not influence expression of the CviI/R QS system or vice versa. VioS furthermore does not significantly affect the levels of AHLs.

To further understand the opposing regulatory effects of VioS and CviR-AHL on violacein production we monitored the reporter activity of a plasmid vioA::gfp transcriptional fusion in the wild type, MB8, MB11, 31532VIOS, cviI, and cviR mutants (Figure 2C). The vioA promoter controls the expression of the operon (vioA-vioE) encoding for the violacein biosynthesis genes (August et al., 2000; Antônio and Creczynski-Pasa, 2004; Sánchez et al., 2006). As expected, little expression of vioA::gfp was apparent in the $c v i I$ and $c v i R$ mutants compared with the wild type. On the other hand vioA::gfp fusion showed a drastic increase in expression in all three vioS mutants, MB8, MB11, and 31532VIOS compared with the wild type

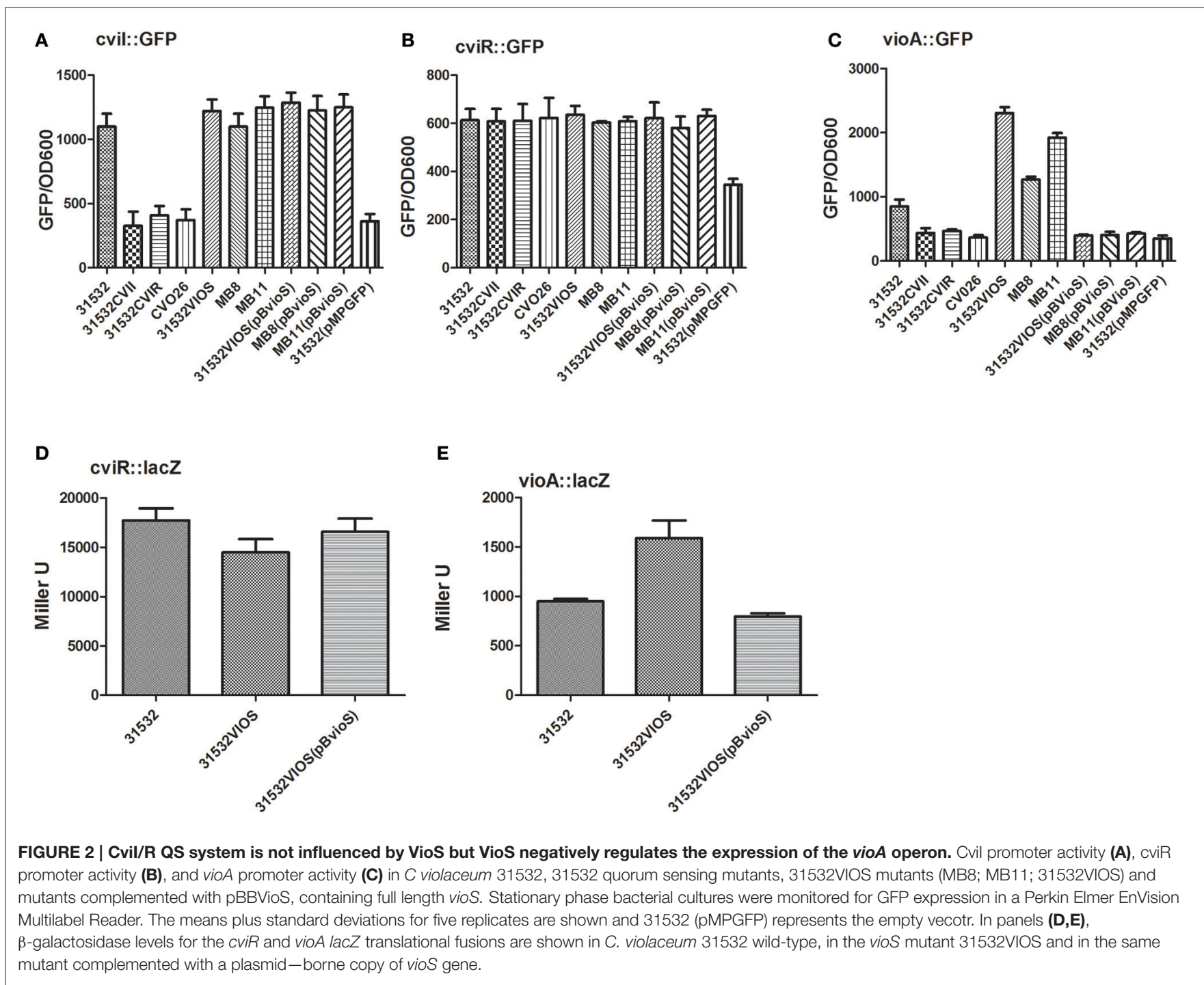


strain. Complementation of the vioS mutants with a wild type copy of the vioS gene restored vioA::gfp expression to wild type levels (Figure 2C). These results demonstrate that VioS represses expression of the vio operon at the transcriptional level thus influencing violacein production in the C. violaceum ATCC31532 wild type strain in spite of presence a functional CviI/R QS system.

To investigate whether VioS has an effect on the translational levels of $c v i I$ and vioA, we constructed $c v i R$-lacZ and vioAlac $Z$ translational fusions as described in the Materials and Methods. As depicted in Figures 2D,E, VioS did not affect $c v i R$ translation. However, in the vioS mutant, the vioAlac $Z$ translational fusion displayed a 2 -fold increase in $\beta$ galactosidase activity. These data indicate that VioS exerts a negative effect on the translation of vio $A$ meaning that it could be acting at a post-transcriptional level; however this increase in translation could be due to the increase in transcription observed using the vioA transcriptional fusion (Figure 2C).

\section{VioS Is Sufficient to Antagonize CviR-Mediated Regulation of the Violacein Biosynthetic Operon in a Heterologous System}

To determine whether VioS is sufficient to antagonize CviRmediated positive regulation of the vio operon, the entire system consisting of VioS, CviR, and the target promoter vioA::lacZ was reconstructed and introduced into a heterologous $E$. coli strain as described in Materials and Methods (Figure 3A). When the activity of vioA::lacZ fusion was monitored in E. coli in the presence of CviR and C6-HSL, the promoter showed high levels of expression consistent with CviR the positively regulating vioA in the presence of the cognate AHL signal.

The increased vioA::lacZ expression was not observed in the absence of C6-HSL. Upon expression of VioS in the same E. coli strain containing CviR and exogenously added C6-HSL, vioA::lacZ expression was reduced by over 6-fold indicating that VioS antagonizes the action of CviR, repressing vioA promoter activity. This observation in a heterologous system also indicates that VioS alone is sufficient to mediate the negative regulation of the vio $A$ promoter.

It was also of interest to establish whether the negative effect of VioS on transcription of an AHL QS target gene was specific for the CviR regulated vioA promoter. Expression studies were therefore carried out using a different AHL QS system and target promoter. For this experiment we used the Burkholderia cepacia CepI/R system and the cepI target gene. The plasmid cepI::lacZ transcriptional fusion construct was introduced into E. coli harboring plasmids expressing either CepR or VioS. The expression of the cepI::lacZ fusion was determined with and without the exogenous addition of C8HSL. In this experiment, the cepI promoter was upregulated in the presence of CepR and AHLs as expected but in contrast to the vioA promoter, it was not repressed in the presence of VioS (Figure 3B). Thus, the VioS mediated effect on the expression of a QS regulated promoter is likely to be specific for the CviI/R system.

\section{QS and VioS Antagonistically Modulate QS-Regulated Phenotypes in C. violaceum}

Since VioS negatively regulates violacein production, we investigated whether it plays a role in fine-tuning the expression
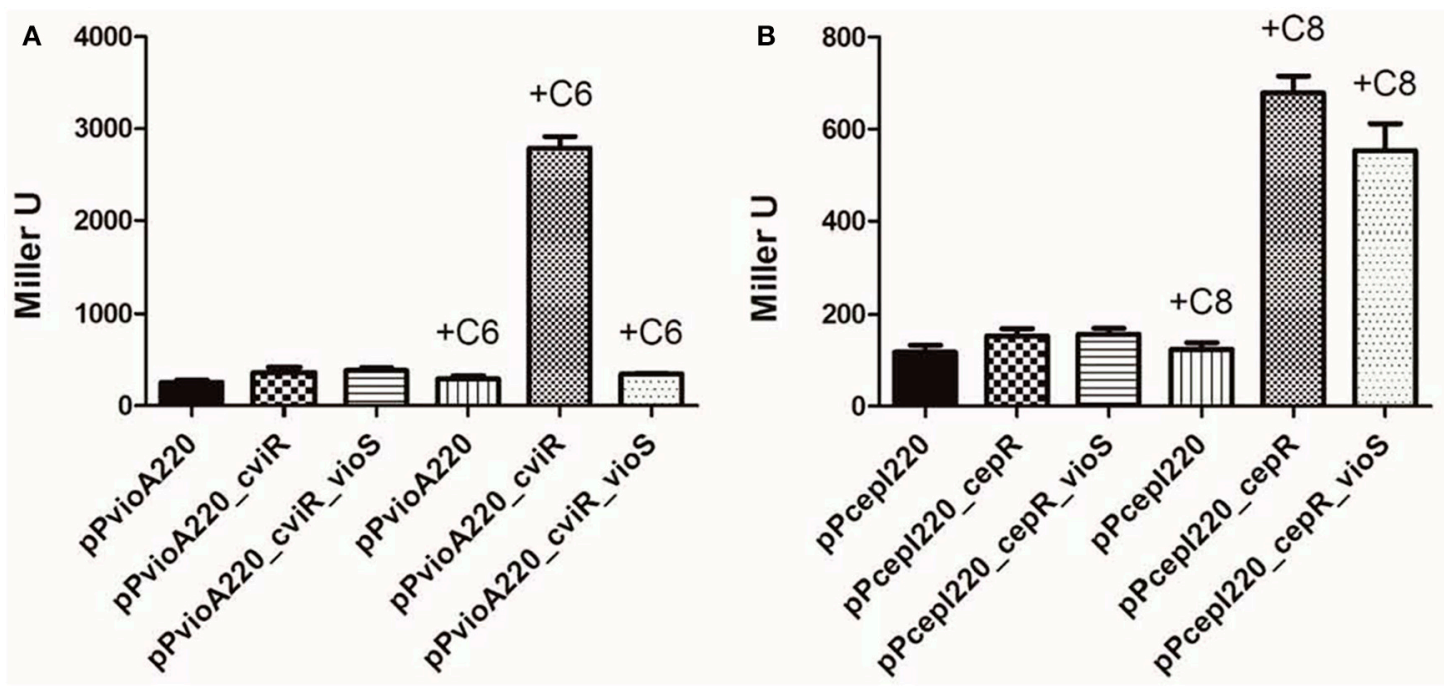

FIGURE 3 | VioS antagonizes CviR mediated activation of the vioA promoter in a heterologous $E$. coli strain and this inhibition is specific. (A) vioA promoter activity in the presence of CViR alone or together with VioS, in the absence/presence of C6-HSL (1 $\mu \mathrm{M})$. (B) cep/ promoter activity in the presence of CepR alone or together with VioS, in the absence/presence of C8-HSL (1 $\mu \mathrm{M})$. $\beta$-gal activities were measured after $12 \mathrm{~h}$ of growth. Experiments were performed in triplicate and means plus standard deviations are plotted. 

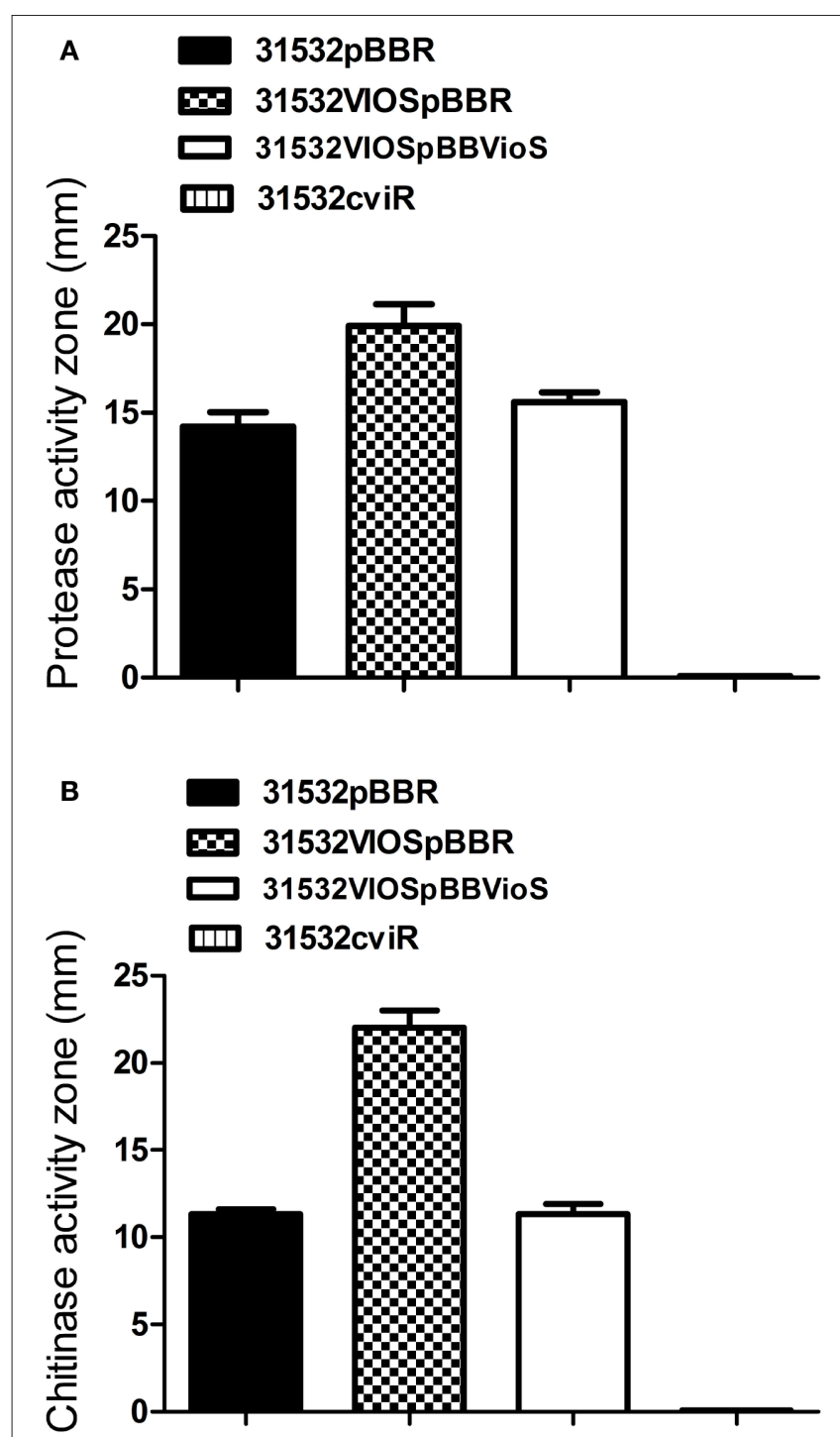

FIGURE 4 | Effect of vioS and cviR mutations on (A) protease activity and (B) chitinase activity. The halos of cleared zones in milk agar plates were measured after $36 \mathrm{~h}$ growth. The halos of cleared zones in colloidal chitin plates were measured after 3 days of incubation. Experiments were performed in triplicate and means plus standard deviations are presented.

of other QS-regulated phenotypes in C. violaceum. Protease and chitinolytic activities are known to be positively regulated by the CviI/R QS system in C. violaceum (Chernin et al., 1998). In the $c v i R$ mutant of ATCC 31532 both protease and chitinase activities were abolished when compared with the wild type. In contrast to this, the vioS mutant showed increased levels of both protease and chitinase activities which could be reduced back to wild type levels by providing VioS in trans (Figures 4A,B). This shows that VioS also acts as a repressor of these two CviI/R QS regulated phenotypes as well as of violacein production. VioS might therefore play a more general role in adjusting the expression of CviI/R QS target genes in a manner opposite to their regulation by CviI/R QS.

\section{DISCUSSION}

In this study we report the regulatory functions of VioS, a putative repressor protein that negatively controls violacein production without influencing expression of the CviI/R QS system. The repressor function of VioS on violacein production is dominant as it antagonizes positive regulation by CviR/C6-HSL in wild type C. violaceum ATCC31532. Other phenotypes positively regulated by CviR-AHL, including protease and chitinase production, were also negatively regulated by VioS. Our results have thus uncovered a novel repressor of C. violaceum QS and identified another layer of population dependent regulation in this bacterium.

C. violaceum is an environmental bacterium, found in soil and water, is generally non-pathogenic but occasionally extremely virulent to humans and animals (Brazilian National Genome Project, 2003). It has been shown that elimination of QS leads to loss of virulence of C. violaceum in a C. elegans model of infection suggesting that functions positively regulated by QS are important for infection (Swem et al., 2009). However, the phenotypes regulated by AHL-dependent QS can be energetically expensive such that constitutive expression of these shared traits is not likely to enable optimal utilization of available resources; it may also elicit stronger host defense responses. RsaL, a negative regulator of QS and QS-regulated genes in Pseudomonas aeruginosa has been reported to be important for optimum virulence as $r s a L$ mutants are hypervirulent in a Galleria mellonella acute model of infection (Rampioni et al., 2009). Also, in a study involving dual-species coculture of $C$. violaceum and Burkholderia thailandensis, it was reported that QS dependent antimicrobials like violacein can provide a competitive advantage in mixed microbial communities with limited nutrients (Chandler et al., 2012). Here, we have shown that VioS functions to fine-tune QSregulated phenotypes and it is possible that it might play a role in providing optimum fitness to $C$. violaceum both in the environment and in host associations. Alternatively, it cannot be excluded that VioS responds to environmental stimuli or an unknown signal that results in de-repression and so promotes high levels of violacein production under certain circumstances.

Although the molecular mechanism of VioS-mediated repression in QS homeostasis is not known, it is possible that it belongs to a new class of regulators. Among the few characterized negative regulators of QS are RsaL, AlgQ, and a TetR-like transcriptional repressor of $P$. aeruginosa, all of which bind DNA (De Kievit et al., 1999; Ledgham et al., 2003; Rampioni et al., 2006; Venturi et al., 2011; Longo et al., 2013). RsaM of $P$. fuscovaginae as well as other repressor proteins with less sequence identity to RsaM including BcRsaM of B. cenocepacia and TofM of B. glumae are also QS repressors (Mattiuzzo et al., 2011; Chen et al., 2012; Michalska et al., 2014). However, BcRsaM is predicted to influence QS by an as yet unknown mechanism but not by binding to DNA (Michalska et al., 2014). The VioS amino acid sequence does not show similarity to any of these proteins and furthermore this study does not provide any direct evidence that VioS exerts its regulation at 
the transcriptional level. Studies performed using translational fusions indicate that VioS had a negative effect on the translation of vioA. A comparison with RsaL of $P$. aeruginosa suggests that VioS exhibits some common and distinct features. The $r s a L$ gene is genetically linked to QS systems and its transcription is positively regulated by QS. However, RsaL negatively regulates expression of lasI coding for AHL synthase as well as some other QS regulated genes responsible for e.g., pyocyanin and HCN production (Schuster et al., 2004; Rampioni et al., 2006, 2007b). RsaL and LasR have been shown to bind to adjacent sites on the lasI promoter but the negative regulatory effect of RsaL is dominant over the activating effect of LasR-AHL (Rampioni et al., 2007a). In our study, the presence of VioS influences vioA promoter activity in a manner similar to RsaL-mediated repression of the lasI promoter because the repressor activity of VioS on vioA promoter is dominant over the activator effect of CviR-AHL. However, unlike the rsaL system where the expression of the repressor is dependent on LasR-AHL, vioS expression is not linked to CviR-AHL and the mechanism of vioS expression and regulation requires further investigation. In addition vioS is found in a separate genomic location from the $c v i I$ and $c v i R$ genes and does not have any direct effect on their transcription but impacts at an as yet unknown level on CviI/R QS target gene expression. Moreover, VioS appears to be sufficient and specific for CviR-AHL antagonism as it is not a general inhibitor of gene activation by other QS LuxR regulators in other bacteria, for example CepR-AHL from Burkholderia.

Sequence similarity searches with the predicted amino acid sequence of C. violaceum ATCC31532 VioS were undertaken to identify homologs of this protein in other bacteria. In our searches VioS homolog was identified only in the sequenced genomes of C. violaceum ATCC12472 strain and P. ferrooxidans. The exclusive presence of VioS in these two bacterial genera suggests that it may have specific functions in these bacterial species. In contrast, other QS repressors like RsaL and RsaM are present in multiple members of the proteobacteria (Venturi et al., 2011). Both C. violaceum and $P$. ferrooxidans produce the purple violacein pigment and it will be interesting to determine whether VioS also regulates pigment production in P. ferrooxidans. These two bacteria could share a similar niche(s) [P. ferrooxidans producing violacein has been isolated in a lake, $\{$ (Puranik, 2013) $\# 736\}]$ as well as profile and regulation of secondary metabolite production in order to survive in specific environmental conditions; this possibility is currently unknown. According to our experiments, the repressor function of VioS for violacein production is conserved in both C. violaceum ATCC31532 and the sequenced strain, C. violaceum ATCC12472 which however differ in the levels of violacein produced. We therefore decided to introduce the vioS gene of strain ATCC31532 in trans into the C. violaceum ATCC12472 wild type and this resulted in the transformation of the deep purple colony color to pale white color (Figure 1) indicative of violacein repression. Interestingly, C. violaceum ATCC12472 wild type has a gene homologous to vioS (CV_1055) and further experiments will be necessary to determine whether this genes codes for a functional protein or has lower expression levels than required to mediate its repressor effect in the presence of CviR-AHL. Interestingly, a very recent study has reported violacein production in the marine bacterium Pseudomonas ulvae and its regulation by AHL QS (Mireille Ayé et al., 2015). It would be interesting to determine whether VioS is present and regulates violacein production in this marine bacterium.

Our current understanding of VioS mediated regulation of violacein biosynthesis in C. violaceum is shown in the schematic model (Figure 5). Briefly, at high cell densities, the CviR protein binds AHLs to activate expression of vioA promoter in C. violaceum wild type. Expression of VioS under these conditions leads to repression of vioA promoter and consequently of violacein production and pale colonies of wild

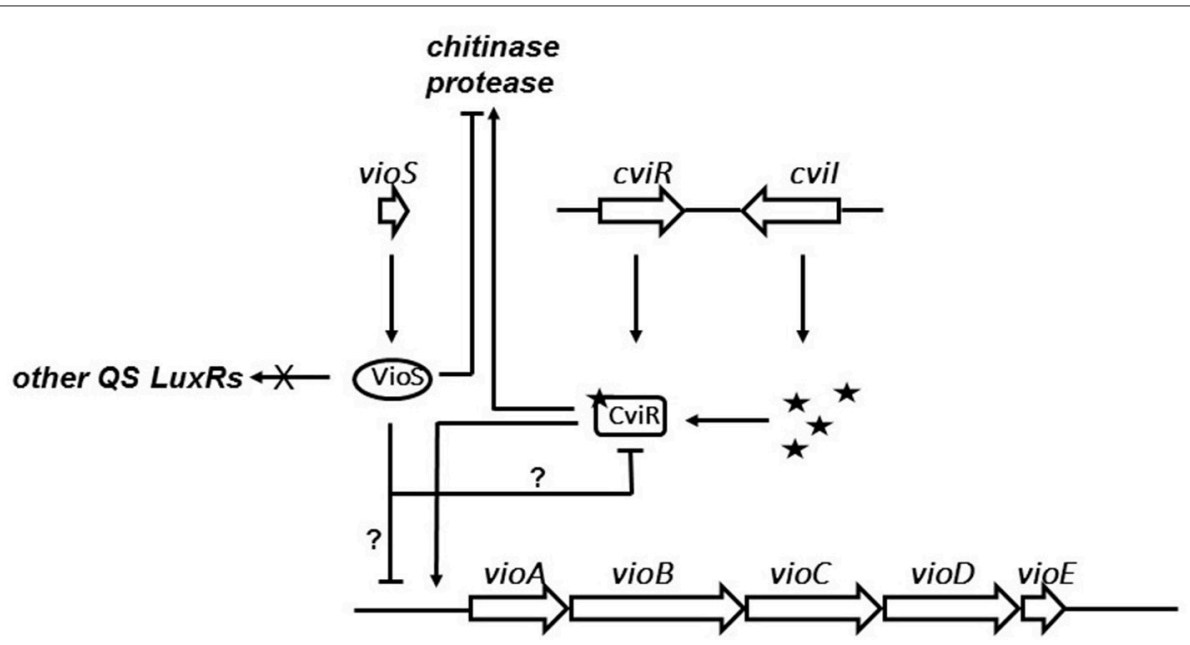

FIGURE 5 | Model for role of VioS in regulation of QS regulated phenotypes in C. violaceum. VioS negatively regulates the vio operon either directly or indirectly, which is positively regulated directly by the Cvil/R QS system. VioS negatively regulates chitinase and protease production, which are positively regulated by the Cvil/R system. 
type C. violaceum ATCC31532. A vioS mutant is relieved from this repression at the vioA promoter leading to violacein production which is clearly visible as purple-colored colonies. Future studies need to address whether the effect of VioS on the vioA promoter is due to a transcriptional, post-transcriptional control or possibly via protein-protein interaction with the CviRAHL complex. In addition from this study it is important to determine the levels of VioS required to antagonize CviR$\mathrm{AHL}$ and the conditions that regulate vioS expression in C. violaceum.

\section{REFERENCES}

Aguilar, C., Bertani, I., and Venturi, V. (2003). Quorum-sensing system and stationary-phase sigma factor (rpoS) of the onion pathogen Burkholderia cepacia genomovar I type strain, ATCC 25416. Appl. Environ. Microbiol. 69, 1739-1747. doi: 10.1128/AEM.69.3.1739-1747.2003

Ahmadian, G., Degrassi, G., Venturi, V., Zeigler, D. R., Soudi, M., and Zanguinejad, P. (2007). Bacillus pumilus SG2 isolated from saline conditions produces and secretes two chitinases. J. Appl. Microbiol. 103, 1081-1089. doi: $10.1111 / j .1365-2672.2007 .03340 . x$

Alexeyev, M. F. (1999). The pKNOCK series of broad-host-range mobilizable suicide vectors for gene knockout and targeted DNA insertion into the chromosome of gram-negative bacteria. BioTechniques 26, 824-826, 828 .

Antônio, R. V., and Creczynski-Pasa, T. B. (2004). Genetic analysis of violacein biosynthesis by Chromobacterium violaceum. Genet. Mol. Res. 3, 85-91.

August, P. R., Grossman, T. H., Minor, C., Draper, M. P., MacNeil, I. A., Pemberton, J. M., et al. (2000). Sequence analysis and functional characterization of the violacein biosynthetic pathway from Chromobacterium violaceum. J. Mol. Microbiol. Biotechnol. 2, 513-519.

Better, M., Lewis, B., Corbin, D., Ditta, G., and Helinski, D. R. (1983). Structural relationships among Rhizobium meliloti symbiotic promoters. Cell 35, 479-485. doi: 10.1016/0092-8674(83)90181-2

Brazilian National Genome Project, C. (2003). The complete genome sequence of Chromobacterium violaceum reveals remarkable and exploitable bacterial adaptability. Proc. Natl. Acad. Sci. U.S.A. 100, 11660-11665. doi: 10.1073/pnas. 1832124100

Chandler, J. R., Heilmann, S., Mittler, J. E., and Greenberg, E. P. (2012). Acylhomoserine lactone-dependent eavesdropping promotes competition in a laboratory co-culture model. ISME J. 6, 2219-2228. doi: 10.1038/ismej.2012.69

Chen, R., Barphagha, I. K., Karki, H. S., and Ham, J. H. (2012). Dissection of quorum-sensing genes in Burkholderia glumae reveals non-canonical regulation and the new regulatory gene tofM for toxoflavin production. PLoS ONE 7:e52150. doi: 10.1371/journal.pone.0052150

Chernin, L. S., Winson, M. K., Thompson, J. M., Haran, S., Bycroft, B. W., Chet, I., et al. (1998). Chitinolytic activity in Chromobacterium violaceum: substrate analysis and regulation by quorum sensing. J. Bacteriol. 180, 4435-4441.

De Kievit, T., Seed, P. C., Nezezon, J., Passador, L., and Iglewski, B. H. (1999). RsaL, a novel repressor of virulence gene expression in Pseudomonas aeruginosa. J. Bacteriol. 181, 2175-2184.

Durán, N., and Menck, C. F. (2001). Chromobacterium violaceum: a review of pharmacological and industiral perspectives. Crit. Rev. Microbiol. 27, 201-222. doi: 10.1080/20014091096747

Figurski, D. H., and Helinski, D. R. (1979). Replication of an origin-containing derivative of plasmid RK2 dependent on a plasmid function provided in trans. Proc. Natl. Acad. Sci. U.S.A. 76, 1648-1652. doi: 10.1073/pnas.76.4.1648

Fuqua, C., and Greenberg, E. P. (2002). Listening in on bacteria: acyl-homoserine lactone signalling. Nat. Rev. Mol. Cell Biol. 3, 685-695. doi: 10.1038/nrm907

Fuqua, C., Winans, S. C., and Greenberg, E. P. (1996). Census and consensus in bacterial ecosystems: the LuxR-LuxI family of quorumsensing transcriptional regulators. Annu. Rev. Microbiol. 50, 727-751. doi: 10.1146/annurev.micro.50.1.727

\section{AUTHOR CONTRIBUTIONS}

GD, MK, SC, and IB performed experimental work whereas MC, PW, SS, and VV drafted the manuscript. All authors were involved in designing, discussing, and interpreting the results of the experiments.

\section{FUNDING}

We thank ICGEB for support.

Fuqua, W. C., Winans, S. C., and Greenberg, E. P. (1994). Quorum sensing in bacteria: the LuxR-LuxI family of cell density-responsive transcriptional regulators. J. Bacteriol. 176, 269-275. doi: 10.1128/jb.176.2.269-275.1994

Kovach, M. E., Elzer, P. H., Hill, D. S., Robertson, G. T., Farris, M. A., Roop, R. M. II, et al. (1995). Four new derivatives of the broad-host-range cloning vector pBBR1MCS, carrying different antibiotic-resistance cassettes. Gene 166, 175-176. doi: 10.1016/0378-1119(95)00584-1

Ledgham, F., Soscia, C., Chakrabarty, A., Lazdunski, A., and Foglino, M. (2003). Global regulation in Pseudomonas aeruginosa: the regulatory protein AlgR2 (AlgQ) acts as a modulator of quorum sensing. Res. Microbiol. 154, 207-213. doi: 10.1016/S0923-2508(03)00024-X

Lichstein, H. C., and Van De Sand, V. F. (1946). The antibiotic activity of violacein, prodigiosin, and phthiocol. J. Bacteriol. 52, 145-146.

Loh, J., Pierson, E. A., Pierson, L. S. III, Stacey, G., and Chatterjee, A. (2002). Quorum sensing in plant-associated bacteria. Curr. Opin. Plant Biol. 5, 285-290. doi: 10.1016/S1369-5266(02)00274-1

Longo, F., Rampioni, G., Bondi, R., Imperi, F., Fimia, G. M., Visca, P., et al. (2013). A new transcriptional repressor of the Pseudomonas aeruginosa quorum sensing receptor gene LasR. PLoS ONE 8:e69554. doi: 10.1371/journal.pone.0069554

Mattiuzzo, M., Bertani, I., Ferluga, S., Cabrio, L., Bigirimana, J., Guarnaccia, C., et al. (2011). The plant pathogen Pseudomonas fuscovaginae contains two conserved quorum sensing systems involved in virulence and negatively regulated by RsaL and the novel regulator RsaM. Environ. Microbiol. 13, 145-162. doi: 10.1111/j.1462-2920.2010.02316.x

McClean, K. H., Winson, M. K., Fish, L., Taylor, A., Chhabra, S. R., Camara, M., et al. (1997). Quorum sensing and Chromobacterium violaceum: exploitation of violacein production and inhibition for the detection of N-acylhomoserine lactones. Microbiology 143 (Pt 12), 3703-3711. doi: $10.1099 / 00221287-143-12-3703$

Michalska, K., Chhor, G., Clancy, S., Jedrzejczak, R., Babnigg, G., Winans, S. C., et al. (2014). RsaM: a transcriptional regulator of Burkholderia spp. with novel fold. FEBS J. 281, 4293-4306. doi: 10.1111/febs. 12868

Miller, J. H. (1972). Experiments in Molecular Genetics. New York, NY: Cold Spring Harbor.

Mireille Ayé, A., Bonnin-Jusserand, M., Brian-Jaisson, F., Ortalo-Magné, A., Culioli, G., Koffi Nevry, R., et al. (2015). Modulation of violacein production and phenotypes associated with biofilm by exogenous quorum sensing $\mathrm{N}$-acylhomoserine lactones in the marine bacterium Pseudoalteromonas ulvae TC14. Microbiology 161, 2039-2051. doi: 10.1099/mic.0.0 00147

Morohoshi, T., Kato, M., Fukamachi, K., Kato, N., and Ikeda, T. (2008). Nacylhomoserine lactone regulates violacein production in Chromobacterium violaceum type strain ATCC 12472. FEMS Microbiol. Lett. 279, 124-130. doi: 10.1111/j.1574-6968.2007.01016.x

O’Toole, G. A., and Kolter, R. (1998). Initiation of biofilm formation in Pseudomonas fluorescens WCS365 proceeds via multiple, convergent signalling pathways: a genetic analysis. Mol. Microbiol. 28, 449-461. doi: 10.1046/j.1365-2958.1998.00797.x

Passos da Silva, D., Castañeda-Ojeda, M. P., Moretti, C., Buonaurio, R., Ramos, C., and Venturi, V. (2014). Bacterial multispecies studies and microbiome 
analysis of a plant disease. Microbiology 160, 556-566. doi: 10.1099/mic.0.07 4468-0

Rampioni, G., Bertani, I., Zennaro, E., Polticelli, F., Venturi, V., and Leoni, L. (2006). The quorum-sensing negative regulator RsaL of Pseudomonas aeruginosa binds to the lasI promoter. J. Bacteriol. 188, 815-819. doi: 10.1128/JB.188.2.815-819.2006

Rampioni, G., Polticelli, F., Bertani, I., Righetti, K., Venturi, V., Zennaro, E., et al. (2007a). The Pseudomonas quorum-sensing regulator rsal belongs to the tetrahelical superclass of H-T-H proteins. J. Bacteriol. 189, 1922-1930. doi: 10.1128/JB.01552-06

Rampioni, G., Schuster, M., Greenberg, E. P., Bertani, I., Grasso, M., Venturi, V., et al. (2007b). RsaL provides quorum sensing homeostasis and functions as a global regulator of gene expression in Pseudomonas aeruginosa. Mol. Microbiol. 66, 1557-1565. doi: 10.1111/j.1365-2958.2007.06029.x

Rampioni, G., Schuster, M., Greenberg, E. P., Zennaro, E., and Leoni, L. (2009). Contribution of the RsaL global regulator to Pseudomonas aeruginosa virulence and biofilm formation. FEMS Microbiol. Lett. 301, 210-207. doi: 10.1111/j.1574-6968.2009.01817.x

Sambrook, J., Fritsch, E. F., and Maniatis, T. (1989). Molecular Cloning: a Laboratory Manual, 2nd Edn. New York, NY: Cold Spring Harbor.

Sánchez, C., Braña, A. F., Méndez, C., and Salas, J. A. (2006). Reevaluation of the violacein biosynthetic pathway and its relationship to indolocarbazole biosynthesis. Chembiochem 7, 1231-1240. doi: 10.1002/cbic.200600029

Schuster, M., Urbanowski, M. L., and Greenberg, E. P. (2004). Promoter specificity in Pseudomonas aeruginosa quorum sensing revealed by DNA binding of purified LasR. Proc. Natl. Acad. Sci. U.S.A. 101, 15833-15839. doi: 10.1073/pnas.0407229101

Simon, R., Priefer, U., and Puhler, A. (1983). A broad host range mobilization system for in vivo genetic engineering: transposon mutagenesis in Gram negative bacteria. Nat. Biotechnol. 1, 784-791. doi: 10.1038/nbt1183-784

Spaink, H. P., Okker, R. J. H., Wijffelmann, C. A., Pees, E., and Lugtemberg, B. J. J. (1987). Promoter in the nodulation region of the Rhizobium leguminosarum Sym plasmid pRL1JI. Plant Mol. Biol. 9, 27-39. doi: 10.1007/BF000 17984

Stachel, S. E., An, G., Flores, C., and Nester, E. W. (1985). A Tn3 lacZ transposon for the random generation of b-galactosidase gene fusions: application to the analysis of gene expression in Agrobacterium. EMBO J. 4, 891-898.

Staskawicz, B., Dahlbeck, D., Keen, N., and Napoli, C. (1987). Molecular characterization of cloned avirulence genes from race 0 and race 1 of Pseudomonas syringae pv. glycinea. J. Bacteriol. 169, 5789-5794. doi: $10.1128 /$ jb.169.12.5789-5794.1987
Stauff, D. L., and Bassler, B. L. (2011). Quorum sensing in Chromobacterium violaceum: DNA recognition and gene regulation by the CviR receptor. J. Bacteriol. 193, 3871-3878. doi: 10.1128/JB.05125-11

Steindler, L., and Venturi, V. (2007). Detection of quorum-sensing N-acyl homoserine lactone signal molecules by bacterial biosensors. FEMS Microbiol. Lett. 266, 1-9. doi: 10.1111/j.1574-6968.2006.00501.x

Swem, L. R., Swem, D. L., O’loughlin, C. T., Gatmaitan, R., Zhao, B., Ulrich, S. M., et al. (2009). A quorum-sensing antagonist targets both membrane-bound and cytoplasmic receptors and controls bacterial pathogenicity. Mol. Cell 35, 143-153. doi: 10.1016/j.molcel.2009.05.029

Swift, S., Karlyshev, A. V., Fish, L., Durant, E. L., Winson, M. K. Chhabra, S. R., et al. (1997). Quorum sensing in Aeromonas hydrophila and Aeromonas salmonicida: identification of the LuxRI homologs AhyRI and AsaRI and their cognate $\mathrm{N}$-acylhomoserine lactone signal molecules. J. Bacteriol. 179, 5271-5281. doi: 10.1128/jb.179.17.5271-528 1.1997

Venturi, V., Rampioni, G., Pongor, S., and Leoni, L. (2011). The virtue of temperance: built-in negative regulators of quorum sensing in Pseudomonas. Mol. Microbiol. 82, 1060-1070. doi: 10.1111/j.1365-2958.2011.07890.x

Von Bodman, S. B., Bauer, W. D., and Coplin, D. L. (2003). Quorum sensing in plant-pathogenic bacteria. Annu. Rev. Phytopathol. 41, 455-482. doi: 10.1146/annurev.phyto.41.052002.095652

Waters, C. M., and Bassler, B. L. (2005). Quorum sensing: cell-to-cell communication in bacteria. Annu. Rev. Cell Dev. Biol. 21, 319-346. doi: 10.1146/annurev.cellbio.21.012704.131001

Winson, M. K., Swift, S., Fish, L., Throup, J. P., Jørgensen, F., Chhabra, S. R., et al. (1998). Construction and analysis of luxCDABE-based plasmid sensors for investigating $\mathrm{N}$-acyl homoserine lactone-mediated quorum sensing. FEMS Microbiol. Lett. 163, 185-192. doi: 10.1111/j.1574-6968.1998.tb13044.x

Conflict of Interest Statement: The authors declare that the research was conducted in the absence of any commercial or financial relationships that could be construed as a potential conflict of interest.

Copyright (C) 2017 Devescovi, Kojic, Covaceuszach, Cámara, Williams, Bertani, Subramoni and Venturi. This is an open-access article distributed under the terms of the Creative Commons Attribution License (CC BY). The use, distribution or reproduction in other forums is permitted, provided the original author(s) or licensor are credited and that the original publication in this journal is cited, in accordance with accepted academic practice. No use, distribution or reproduction is permitted which does not comply with these terms. 